QUARTERLY OF APPLIED MATHEMATICS

VOLUME LXVII, NUMBER 2

JUNE 2009, PAGES 265-281

S 0033-569X(09)01122-2

Article electronically published on March 19, 2009

\title{
NONLINEAR INTERFACIAL WAVES IN STREAMING FLOWS
}

\author{
BY \\ KADRY ZAKARIA \\ Department of Mathematics, Faculty of Science, Tanta University, Tanta, Egypt
}

\begin{abstract}
The nonlinear interfacial waves between viscous immiscible liquids have been analyzed, using the concepts of viscous potential flow and Kelvin-Helmholtz instability. The method of multiple scales is used for determining the evolution equations that are near and on the marginal state of the linear theory. We use the modulation concept in solving these equations to determine the stability criteria. Different numerical examples are considered that show the system is at greater risk of instability when the velocity of the stream is larger, whereas the effects of viscosity can be stabilizing or destabilizing.
\end{abstract}

1. Introduction. We know from Joseph et al. [1, 2, 3, 4, 5, 6, 7, that the NavierStokes equations are satisfied by potential flow; the viscous term is not found when the vorticity is zero, but the viscous stresses are not zero. The viscous stresses enter into the viscous potential flow analysis of free surface problems through the normal stress balance at the interface. Using viscous potential flow analysis, Joseph et al. have given good approximations to fully viscous flows. Joseph, Belanger and Beavers 3 constructed a viscous potential flow analysis of the Rayleigh-Taylor instability. The normal stress is an extensional rather than a shear stress and it is activated by waves on the liquid; these waves are induced more by pressure than shear. For this reason, we can conclude that the neglect of shearing stress can be justified in wave motions in which the viscous resistance to wave motion is not negligible; this is a situation which may be well approximated by viscous potential flow. Joseph and Liao [1, 2] gave a review for the theory of viscous potential flow. Funada and Joseph [4 studied the viscous potential flow analysis of Kelvin-Helmholtz instability in a channel and they gave a linear study for the problem in an extended form. Their results match with the experiments. The growth rates of the fully viscous fluid analysis and viscous potential flow are matched. The success of viscous potential flow theory in the analysis of Rayleigh-Taylor instability has led to its use in the analysis of Kelvin-Helmholtz (KH) theory (Funada and Joseph 44) given in this work. Wang and Joseph 7] constructed purely irrotational theories of the Stokes problem which are in good agreement with Lamb's exact solution.

Received October 28, 2007.

2000 Mathematics Subject Classification. Primary 34C15.

(C)2009 Brown University

Reverts to public domain 28 years from publication 
This work deals with the study of the viscous potential flow analysis of nonlinear $\mathrm{KH}$ instability for two superposed immiscible fluids near and on the curve of the marginal state of linear theory. The viscosity in viscous potential flow enters into the normal stress balance rather than the tangential stress balance.

Many investigators, such as Hasimoto and Ono [8], Nayfeh 9], Newton and Keller [10] and Zakaria [11, 12] examined the linear stability of a finite-amplitude wave train propagating through the interface. They perturbed the solution of the evolution equations linearly. These equations are the solvability conditions of the applied method of solution. This concept of solution is called the modulation method. In this work we use this concept to study the stability criteria of the interfacial waves.

In the following section, the definition of the considered problem has been shown. In section 3, the solvability conditions of the method of multiple scales have been obtained. In section 4, the linear solution, the neutral curve and a numerical discussion have been examined. Stability analysis of modulated waves near and on the curve of the marginal state and numerical applications, which will show the stability criteria, have been introduced in section 5 . The results have been summarized in section 6 .

2. Formulation of the problem. Consider two viscous incompressible immiscible streaming fluids. The motion is limited to two dimensions $(x, y)$, where $x$ is aligned with the mean interface level, and it is assumed to be represented by potential functions (according to the viscous potential flow theory), as in Fig. 1. The distance and the time are made dimensionless using $d=\left(T / g \rho_{1}\right)$ and $\sqrt{d / g}$ respectively, where $g$ is gravity assumed to be acting along the negative $y$-axis and $T$ is surface tension. The quantities $\rho_{1}$ and $\rho_{2}$ are the densities of the lower $(-\infty<y<0)$ and upper fluids $(0<y<\infty)$, respectively. The velocity potential function $\phi_{j}$ is made dimensionless by $\sqrt{g d^{3}}$. The basic equations that govern the perturbed velocity potential $\phi_{j}$ with their boundary

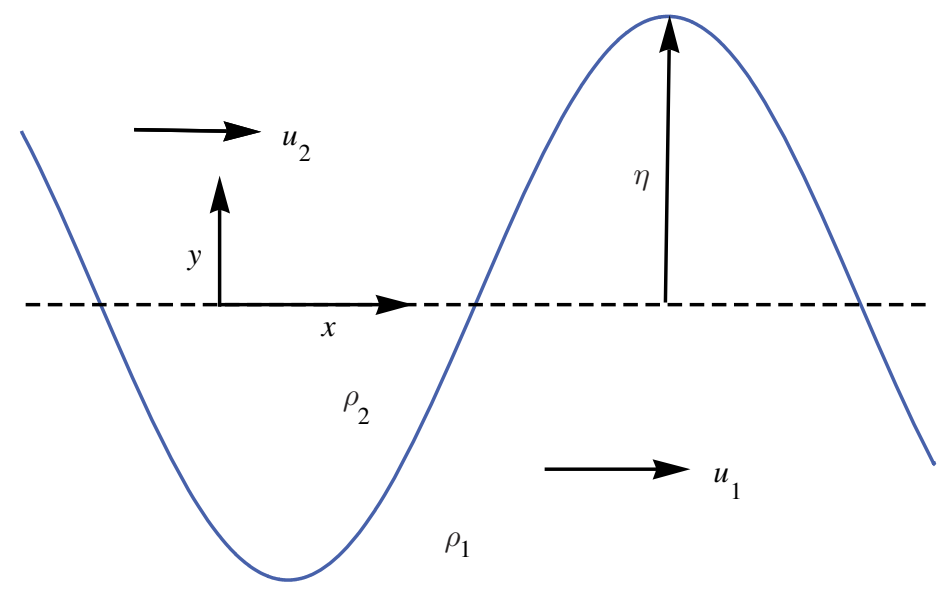

FIG. 1. Sketch of the system under consideration. 
conditions are:

$$
\begin{gathered}
\nabla^{2} \phi_{j}=0, j=1,2, \\
\frac{\partial \phi_{j}}{\partial y}=\frac{\partial \eta}{\partial t}+u_{j} \frac{\partial \eta}{\partial x}+\frac{\partial \eta}{\partial x} \frac{\partial \phi_{j}}{\partial x}, y=\eta, j=1,2 \\
\sum_{j=1}^{2}(-\rho)^{j-1}\left(\frac{\partial \phi_{j}}{\partial t}+\frac{1}{2}\left(\nabla \phi_{j}\right)^{2}+\eta+u_{j} \frac{\partial \phi_{j}}{\partial x}\right) \\
+2(-1)^{j-1} \hat{\mu}_{\ell j}\left(\left(\frac{\partial \eta}{\partial x}\right)^{2} \frac{\partial^{2} \phi_{j}}{\partial x^{2}}+\frac{\partial^{2} \phi_{j}}{\partial y^{2}}-2 \frac{\partial \eta}{\partial x} \frac{\partial^{2} \phi_{j}}{\partial x \partial y}-\left(\frac{\partial \eta}{\partial x}\right)^{2} \frac{\partial^{2} \phi_{j}}{\partial y^{2}}\right) \\
-\left(1-\frac{3}{2}\left(\frac{\partial \eta}{\partial x}\right)^{2}\right) \frac{\partial^{2} \eta}{\partial x^{2}}=0, \quad y=\eta
\end{gathered}
$$

where $\rho=\rho_{2} / \rho_{1}$ and $\hat{\mu}_{\ell j}=\nu_{j} \sqrt{g d} / T$, since $\nu_{j}$ is the viscosity coefficient. We expand all the boundary conditions at the interface $y=\eta(x, t)$ about $y=0$ using Maclaurin's series.

3. The method of solution and evolution equations. To investigate the nonlinear finite interfacial waves, we use the multiple scales method in the time $t$ and the dimension $x$, since $t_{0}=t, t_{1}=\varepsilon t, t_{2}=\varepsilon^{2} t, x_{0}=x, x_{1}=\varepsilon x$ and

$$
f(x, y, t ; \varepsilon)=\sum_{\ell=1}^{3} \varepsilon^{\ell} f_{\ell}\left(x_{0}, x_{1}, y, t_{0}, t_{1}, t_{2}\right)+O\left(\varepsilon^{4}\right)
$$

where $f(x, y, t ; \varepsilon)$ is called $\phi$ or $\eta$. The parameter $\epsilon$ represents a small dimensionless parameter characterizing the steepness ratio of the wave.

Using the scales of the time $t$ and dimension $x$, and inserting equation (4) into equations (1)-(3), equating coefficients of equal powers in $\varepsilon$, we obtain the linear as well as the successive higher-order equations. The equations of the first-order, second-order and third-order are given in the Appendix.

The solution of the first-order equation of the approximation (the linear theory), given by

$$
\phi_{j 1}=\varepsilon \frac{i(-1)^{j}\left(\omega-k u_{j}\right)}{k} A\left(t_{1}, t_{2}, x_{1}\right) e^{i\left(k x_{0}-\omega t_{0}\right)} e^{(-1)^{j+1} k y}+\mathrm{cc},
$$

where the linear elevation is given by

$$
\eta_{1}=\varepsilon A\left(t_{1}, t_{2}, x_{1}\right) e^{i\left(k x_{0}-\omega t_{0}\right)}+\mathrm{cc}
$$

and $A\left(t_{1}, t_{2}, x_{1}\right)$ is the amplitude of the finite amplitude wave, will be studied in the following sections. The term cc refers to the complex conjugate of the previous terms in the right-hand side of the same equation. 
Let us substitute the first-order solutions into the equations of order $\varepsilon^{2}$. The uniformly valid solutions of the resulting equations depend on removing the following secular term:

$$
\begin{aligned}
\left(-\frac{2 i \omega(1+\rho)}{k}\right. & \left.+2 i u_{1}+2 i \rho u_{2}+2 k \hat{\mu}_{\ell 1}+2 k \hat{\mu}_{\ell 2}\right) \frac{\partial A}{\partial t_{1}} \\
& +\left(i u_{1}^{2}+i \rho u_{2}^{2}-2 i k-i \frac{\omega^{2}(1+\rho)}{k^{2}}\right. \\
& \left.-2 \omega \hat{\mu}_{\ell 1}+4 k u_{1} \hat{\mu}_{\ell 1}-2 \omega \hat{\mu}_{\ell 2}+4 k u_{2} \hat{\mu}_{\ell 2}\right) \frac{\partial A}{\partial x_{1}}=0
\end{aligned}
$$

and so the second-order solutions are given by

$$
\begin{aligned}
\eta_{2}= & \frac{i\left(k\left(k^{2}+1+\rho\right)\left(\rho \hat{\mu}_{\ell 1}^{2}-\hat{\mu}_{\ell 2}^{2}\right)\left(u_{1}-u_{2}\right)+4 k^{2} \hat{\mu}_{\ell 1} \hat{\mu}_{\ell 2}\left(k^{2}+1-\rho\right)\left(\hat{\mu}_{\ell 1}+\hat{\mu}_{\ell 2}\right)\right)}{\left(2 k^{2}-1+\rho\right)\left(\rho \hat{\mu}_{\ell 1}^{2}+\hat{\mu}_{\ell 2}^{2}\right)} \\
& \times A^{2} e^{2 i\left(k x_{0}-\omega t_{0}\right)}+\mathrm{cc}
\end{aligned}
$$

and

$$
\begin{aligned}
\phi_{j 2}= & -\left\{\frac{1}{k}(-1)^{j} \frac{\partial A}{\partial t_{1}}+\frac{1}{k^{2}}\left[\left((-1)^{j}+k y\right) \omega-k^{2} u_{j} y\right] \frac{\partial_{A}}{\partial x_{1}}\right\} e^{i\left(k x_{0}-\omega t_{0}\right)} e^{(-1)^{j+1} k y} \\
& +\frac{\left[(-1)^{j+1} Q_{i}+i\left(k+(-1)^{j} Q_{r}\right)\right]\left(\omega-k u_{j}\right)}{k^{2}} e^{2 i\left(k x_{0}-\omega t_{0}\right)} e^{2(-1)^{j+1} k y}+\mathrm{cc},
\end{aligned}
$$

where

$$
Q_{i}=\frac{4 k^{2} \hat{\mu}_{\ell 1} \hat{\mu}_{\ell 2}\left(k^{2}+1-\rho\right)\left(\hat{\mu}_{\ell 1}+\hat{\mu}_{\ell 2}\right)}{\left(2 k^{2}-1+\rho\right)\left(\rho \hat{\mu}_{\ell 1}^{2}+\hat{\mu}_{\ell 2}^{2}\right)}, Q_{r}=\frac{k\left(k^{2}+1+\rho\right)\left(\rho \hat{\mu}_{\ell 1}^{2}-\hat{\mu}_{\ell 2}^{2}\right)\left(u_{1}-u_{2}\right)}{\left(2 k^{2}-1+\rho\right)\left(\rho \hat{\mu}_{\ell 1}^{2}+\hat{\mu}_{\ell 2}^{2}\right)} .
$$

Substituting the first- and second-order solutions into the third-order equations, we have the following solvability condition:

$$
\begin{aligned}
& \frac{1}{k^{2}}\left(i k u_{1}+i k \rho u_{2}-i \omega-i \rho \omega+k^{2} \hat{\mu}_{\ell 1}+k^{2} \hat{\mu}_{\ell 2}\right) \frac{\partial A}{\partial t_{2}} \\
& \quad+\frac{1}{2 k^{2}}\left(\omega+\rho \omega-i k^{2} \hat{\mu}_{\ell 1}-k^{2} \hat{\mu}_{\ell 2}\right) \frac{\partial^{2} A}{\partial t_{1} \partial x_{1}} \\
& \quad+\frac{1}{2 k}(1+\rho) \frac{\partial^{2} A}{\partial t_{1}^{2}}+\frac{1}{2 k^{3}}\left(\omega^{2}+\rho \omega^{2}-k^{3}-2 i k^{3} u_{1} \hat{\mu}_{\ell 1}-2 i k^{3} u_{2} \hat{\mu}_{\ell 2}\right) \frac{\partial^{2} A}{\partial x_{1}^{2}} \\
& \quad+\gamma A^{2} \bar{A}=0,
\end{aligned}
$$

where

$$
\begin{aligned}
\gamma= & \left(-\frac{3 k^{4}}{4}+k \omega^{2}+k \rho \omega+i \omega^{2} Q_{i}-i \rho \omega^{2} Q_{i}+\omega^{2} Q_{r}-\rho \omega^{2} Q_{r}-2 k+k^{2} \omega u_{1}\right. \\
& -2 i k \omega u_{1} Q_{i}-2 k \omega u_{1} Q_{r}+k^{3} u_{1}^{2}+i k^{2} u_{1}^{2} Q_{i}+k^{2} u_{1}^{2} Q_{r}-2 k^{2} \rho \omega u_{2} \\
& +2 i k \rho \omega u_{2} Q_{i}+2 k \rho \omega u_{2} Q_{r}+k^{3} \rho u_{2}^{2}-i k^{2} \rho u_{2}^{2} Q_{i}-k^{2} \rho u_{2}^{2} Q_{r} \\
& \left.+3 i k^{3} \omega\left(\hat{\mu}_{\ell 1}+\hat{\mu}_{\ell 2}\right)-3 i k^{4} u_{1} \hat{\mu}_{\ell 1}-3 i k^{4} u_{2} \hat{\mu}_{\ell 2}\right),
\end{aligned}
$$

4. The curve of the marginal state. In various instability phenomena, the stability of a basic flow is often described by a single dimensionless stability parameter, say the stream velocity or the viscosity number or one of the external forces. If the stability of the basic flow is examined by a linear theory, a neutral curve, or what we call a marginal state curve, then the curves shown in Fig. 2 are obtained. 

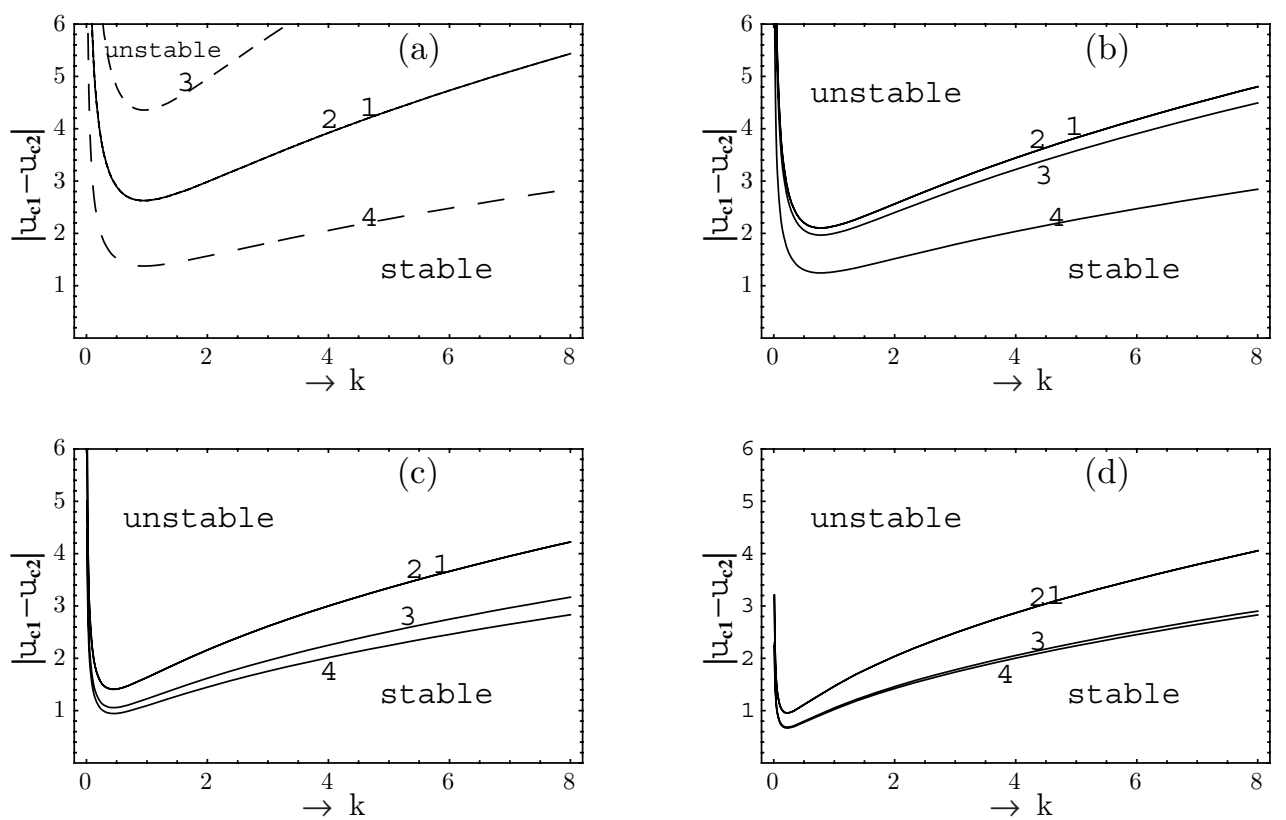

FIG. 2. The curves represent the equation (13). The curves $1,2,3$, and 4 are for $\hat{\mu}_{\ell 1}=0.02$ and $\hat{\mu}_{\ell 2}=0.02, \hat{\mu}_{\ell 1}=4.02$ and $\hat{\mu}_{\ell 2}=4.02$, $\hat{\mu}_{\ell 1}=2.02$ and $\hat{\mu}_{\ell 2}=0$, and $\hat{\mu}_{\ell 1}=0$ and $\hat{\mu}_{\ell 2}=2.02$, respectively: (a) $\rho=0.1$, (b) $\rho=0.4$, (c) $\rho=0.8$, (d) $\rho=0.95$.

Let us replace $-i \omega$ by $\Omega$ to have the following dispersion relation:

$$
A \Omega^{2}+2 B \Omega+C=0,
$$

where $A=1+\rho, B=B_{r}+i B_{i}=k^{2}\left(\hat{\mu}_{\ell 1}+\hat{\mu}_{\ell 2}\right)+i k\left(u_{1}+\rho u_{2}\right)$, and $C=C_{r}+i C_{i}=$ $k^{3}+k-k^{2} u_{1}^{2}-k \rho-k^{2} \rho u_{2}^{2}+2 i k^{3}\left(u_{1} \hat{\mu}_{\ell 1}+u_{2} \hat{\mu}_{\ell 2}\right)$. In general, the frequency $\Omega$ is a complex quantity, so we can put $\Omega=\Omega_{r}+i \Omega_{i}$. To obtain the stability criteria at the marginal state, we must have $\Omega_{r}=0$, and

$$
\Omega_{i}=-\frac{C_{i}}{2 B_{r}}=-\frac{k u_{1} \hat{\mu}_{\ell 1}+k u_{2} \hat{\mu}_{\ell 2}}{\hat{\mu}_{\ell 1}+\hat{\mu}_{\ell 2}},
$$

and this means that

$$
\omega=\frac{k u_{1} \hat{\mu}_{\ell 1}+k u_{2} \hat{\mu}_{\ell 2}}{\hat{\mu}_{\ell 1}+\hat{\mu}_{\ell 2}} .
$$

The transition curve (the marginal state) will be

$$
\left|u_{1}-u_{2}\right|=\left(\hat{\mu}_{\ell 1}+\hat{\mu}_{\ell 2}\right) \sqrt{\frac{1+k^{2}-\rho}{k\left(\hat{\mu}_{\ell 1}^{2}+\rho \hat{\mu}_{\ell 2}^{2}\right)}} .
$$

Equation (14) shows that the case of the two inviscid fluids cannot be treated as a special case. However, equation (14) can only be utilized to investigate the case when one of the two fluids is inviscid while the other is viscous. The influence of the viscosity numbers $\hat{\mu}_{\ell 1}$ and $\hat{\mu}_{\ell 2}$ as well as the effect of the density ratio $\rho$ on the stability criteria, in the linear theory, has been investigated throughout the four frames of Fig. 2. The density 
ratio $\rho$ has acquired the specific values $0.1,0.4,0.5$, and 0.95 , corresponding to the parts (a), (b), (c), and (d) of Fig. 2, respectively. In each section of Fig. 2, we have drawn the transition curve (14), i.e., the curve separating the stable region from the unstable region, in the plane $\left(u_{1}-u_{2}\right)-k$ at four different values of the viscosity numbers for the sake of comparison. The curve indicated by the number (1) represents the transition curve (14) at the values $\hat{\mu}_{\ell 1}=0.02$ and $\hat{\mu}_{\ell 2}=0.02$ and the distinct curve with number (2) represents the same transition curve at the values $\hat{\mu}_{\ell 1}=4.02$ and $\hat{\mu}_{\ell 2}=4.02$, while the curve numbered by (3) is plotted at $\hat{\mu}_{\ell 1}=2.02$ and $\hat{\mu}_{\ell 2}=0$. The curve indicated by number (4) is drawn at $\hat{\mu}_{\ell 1}=0$ and $\hat{\mu}_{\ell 2}=4.02$. Having noted the transition curves displayed in part (a), it is observed that the viscosity coefficients have no influence on the stability criteria of the linear system as these coefficients have the same value. We also note that, when the viscosity number of the upper liquid is decreased, the viscosity number of the lower liquid plays a stabilizing role. On the contrary, we note that, when the viscosity number of the lower liquid is decreased, the viscosity number of the upper liquid plays an unstable role. In other words, the graphs of part (b) show that an increase of the values of the viscosity number of the lower liquid will lead to an increase in the unstable area due to the decrease in the values of the viscosity coefficient of the upper liquid. On the other hand, when the values of the viscosity number of the lower liquid have been decreased, the increase in the viscosity number of the upper liquid plays an unstable role. We remark in parts (b, c) of Fig. 2 that despite the increase of the value of $\rho$ to 0.45 and 0.8 , respectively, the system's behavior resembles exactly the same behavior as in the state (b). However, the unstable role played by both the viscosity number of the above liquid when there is no viscosity number for the lower one and the viscosity number of the lower liquid when there is no viscosity for the higher liquid, decreases with the increase in $\rho$ values.

\section{Stability of modulated waves.}

5.1. Stability of the trivial solution. Near the marginal state we can put $u_{j}=u_{c j}+$ $\epsilon^{2} \Delta_{j}$, since $j=1,2$, and $\Delta_{j}$ is a very small order term. So we have

$$
u_{c 2}=u_{c 1}+\left(\hat{\mu}_{\ell 1}+\hat{\mu}_{\ell 2}\right) \sqrt{\frac{1+k^{2}-\rho}{k\left(\hat{\mu}_{\ell 1}^{2}+\rho \hat{\mu}_{\ell 2}^{2}\right)}}+\Delta, u_{c 2}>u_{c 1},
$$

or

$$
u_{c 1}=u_{c 2}+\left(\hat{\mu}_{\ell 1}+\hat{\mu}_{\ell 2}\right) \sqrt{\frac{1+k^{2}-\rho}{k\left(\hat{\mu}_{\ell 1}^{2}+\rho \hat{\mu}_{\ell 2}^{2}\right)}}+\Delta, u_{c 1}>u_{c 2},
$$

where $\Delta=\Delta_{1}-\Delta_{2}$. We can simplify the equations (7) and (10) to take the following form:

$$
\frac{\partial A}{\partial \zeta}+\left(x_{r 1}+i x_{i 1}\right) \frac{\partial^{2} A}{\partial \xi^{2}}+\left(x_{r 2}+i x_{i 2}\right) A+\left(x_{r 3}+i x_{i 3}\right) A^{2} \bar{A}=0 .
$$

At the marginal state, where

$$
u_{c 1}=u_{c 2}+\left(\hat{\mu}_{\ell 1}+\hat{\mu}_{\ell 2}\right) \sqrt{\frac{1+k^{2}-\rho}{k\left(\hat{\mu}_{\ell 1}^{2}+\rho \hat{\mu}_{\ell 2}^{2}\right)}}, u_{c 1}>u_{c 2},
$$


we have the evolution equation

$$
\frac{\partial A}{\partial \zeta}+\left(x_{r 1}+i x_{i 1}\right) \frac{\partial^{2} A}{\partial \xi^{2}}+\left(x_{r 3}+i x_{i 3}\right) A^{2} \bar{A}=0
$$

where

$$
x_{r 1}=\frac{1+\tilde{\gamma} \tilde{\lambda}}{1+\tilde{\lambda}^{2}}, \quad x_{i 1}=\frac{\tilde{\lambda}-\tilde{\gamma}}{1+\tilde{\lambda}^{2}}, \quad \tilde{\lambda}=\frac{\lambda_{r 1}}{\lambda_{i 1}}, \quad \tilde{\gamma}=\frac{\gamma_{r}}{\gamma_{i}}, \quad x_{r 3}=\frac{\beta_{i}+\tilde{\lambda} \beta_{r}}{1+\tilde{\lambda}^{2}}, \quad x_{i 3}=\frac{\tilde{\lambda} \beta_{i}-\beta_{r}}{1+\tilde{\lambda}^{2}} .
$$

The trivial solution of the evolution equation (17) is $A=0$. To obtain stability of this solution, near the marginal state, we use the concept of modulation [8, 9, 10, 11, 12, so we perturb the steady solution, linearly, to have $A=a(\zeta, \xi)$ for which its evolution is described by

$$
A=a_{1}(\xi, \zeta) e^{i\left(p \xi+b_{1}(\xi, \zeta)\right)},
$$

where $a_{1}$ and $b_{1}$ are linear real functions. Substituting the perturbed form (20) into (17), we have

$$
\varpi=i\left(p^{2} x_{i 1}-x_{i 2}\right)+p^{2} x_{r 1}-x_{r 2} .
$$

To obtain a stable perturbed solution we must have

$$
p^{2}<\left(x_{r 2} / x_{r 1}\right) \text { and } x_{r 1}>0
$$

or

$$
p^{2}>\left(x_{r 2} / x_{r 1}\right) \text { and } x_{r 1}<0 .
$$

The transition curve of those stability criteria is given by $p^{2}=\left(x_{r 2} / x_{r 1}\right)$.

The different parts of Figs. 3-6 in which we study the influences of the viscosity numbers, the stream velocity and the densities of the liquids in the nonlinear stage on the stability criteria near the marginal state are plotted to reflect the stability conditions $(22)$ and $(23)$ in the $\left(p^{2}, k\right)$ plane. The areas which are marked with "S" present the stable areas while those which are marked with "U" indicate the unstable areas. In all of these figures, part (a) is plotted at the values $\hat{\mu}_{\ell 1}=0.02$ and $\hat{\mu}_{\ell 2}=0.02$, part (b) at $\hat{\mu}_{\ell 1}=2.02$ and $\hat{\mu}_{\ell 2}=2.02$, part (c) at $\hat{\mu}_{\ell 1}=0$ and $\hat{\mu}_{\ell 2}=2.02$, and part (d) at $\hat{\mu}_{\ell 1}=2.02$ and $\hat{\mu}_{\ell 2}=0$. We see in Fig. 3(a) that there are two branches of the curves which in turn divide the plane $\left(p^{2}, k\right)$ into three areas: an unstable area that separates two stable areas. The range $k<0.8$ presents a nearly stable area while the range $k>0.8$ is divided into two areas, one of them stable and the other unstable. We note in Fig. 3(b) that there are three branches of the transition curves which divide the plane of stability criteria into four areas, three of them stable and one unstable. In comparing the two parts (a) and (b) of Fig. 3, we remark that although there is an equality of the viscosity numbers in the two parts, the increase of these values in part (b) shows the stable areas and decreases the unstable areas. We see in part (c) that there is a great change in the stable and unstable areas. These areas decreased with the change of some areas from the stable kind to the opposite kind; conversely, with the increase of the areas which divided the $\left(p^{2}, k\right)$ plane in part (b) where $\hat{\mu}_{\ell 1}=2.02$ and $\hat{\mu}_{\ell 2}=0$, we note that there is a very dramatic change in the system's behavior that becomes unstable completely with all values. In the different parts of Fig. 4 the same accounts which are presented in Fig. 3 are repeated but at the values $\rho=0.1, u_{c 2}=2.6$. 

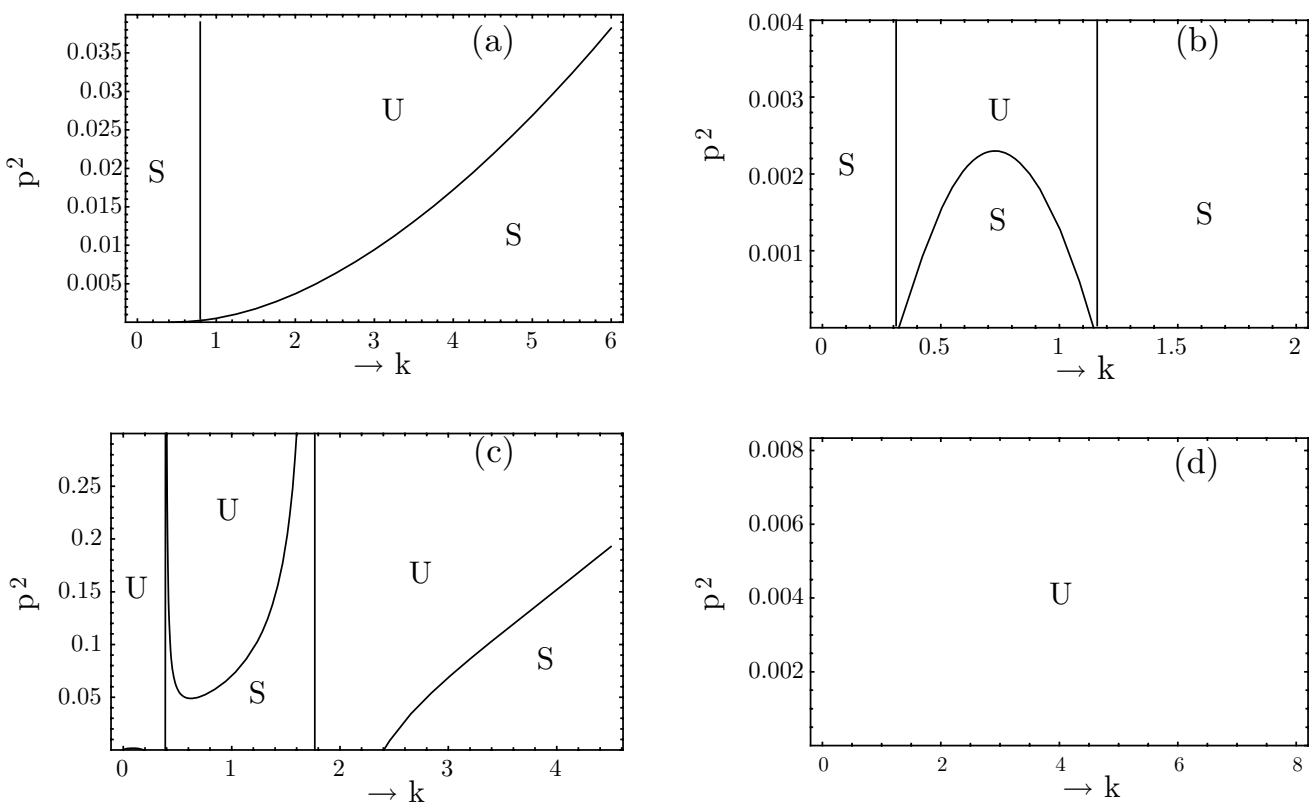

FIG. 3. The curves represent the conditions (21) and (22) for a system having $\rho=0.1$ and $u_{c 2}=0$ : (a) $\hat{\mu}_{\ell 1}=0.02$ and $\hat{\mu}_{\ell 2}=0.02$, (b) $\hat{\mu}_{\ell 1}=2.02$ and $\hat{\mu}_{\ell 2}=2.02$, (c) $\hat{\mu}_{\ell 1}=0$ and $\hat{\mu}_{\ell 2}=0.02$, (d) $\hat{\mu}_{\ell 1}=2.02$ and $\hat{\mu}_{\ell 2}=0$.
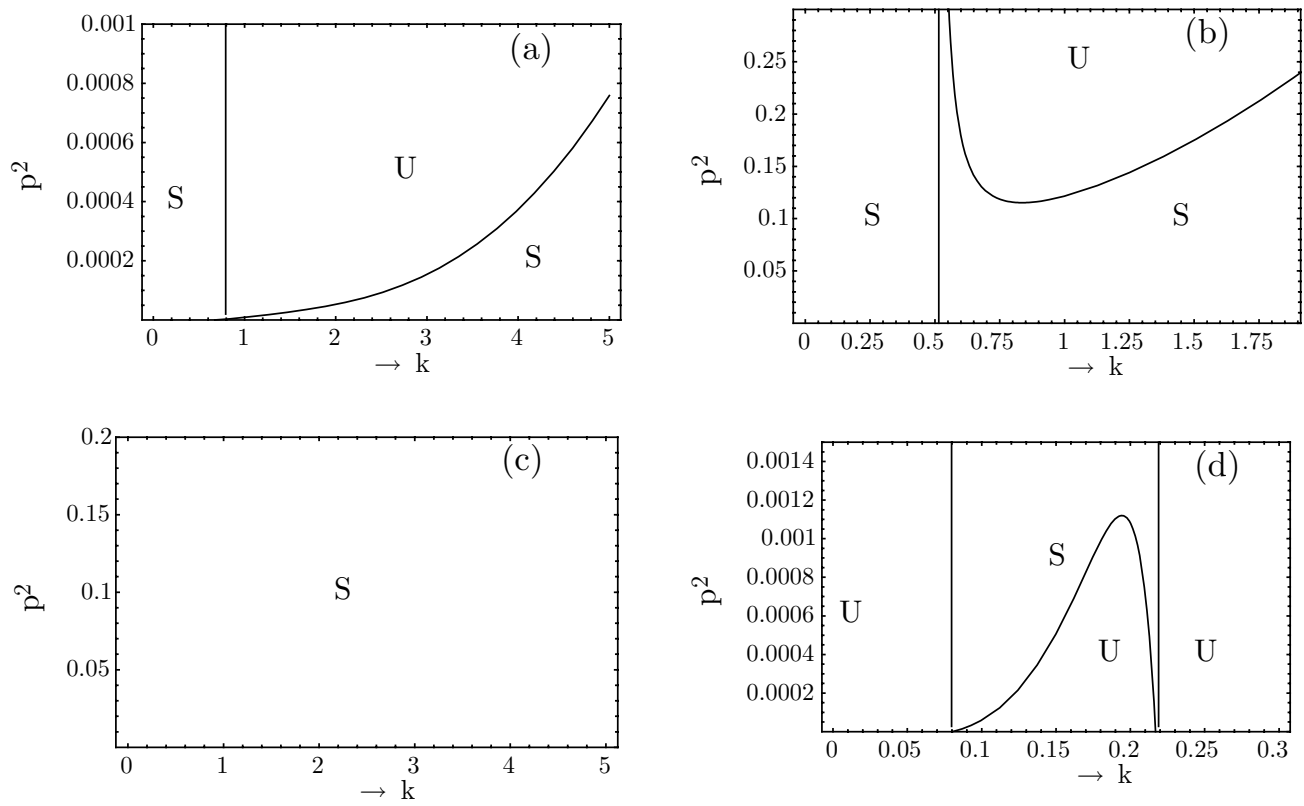

FIG. 4. For the same system considered in Fig. 3 but for $\rho=0.1$ and $u_{c 2}=2.6$. 

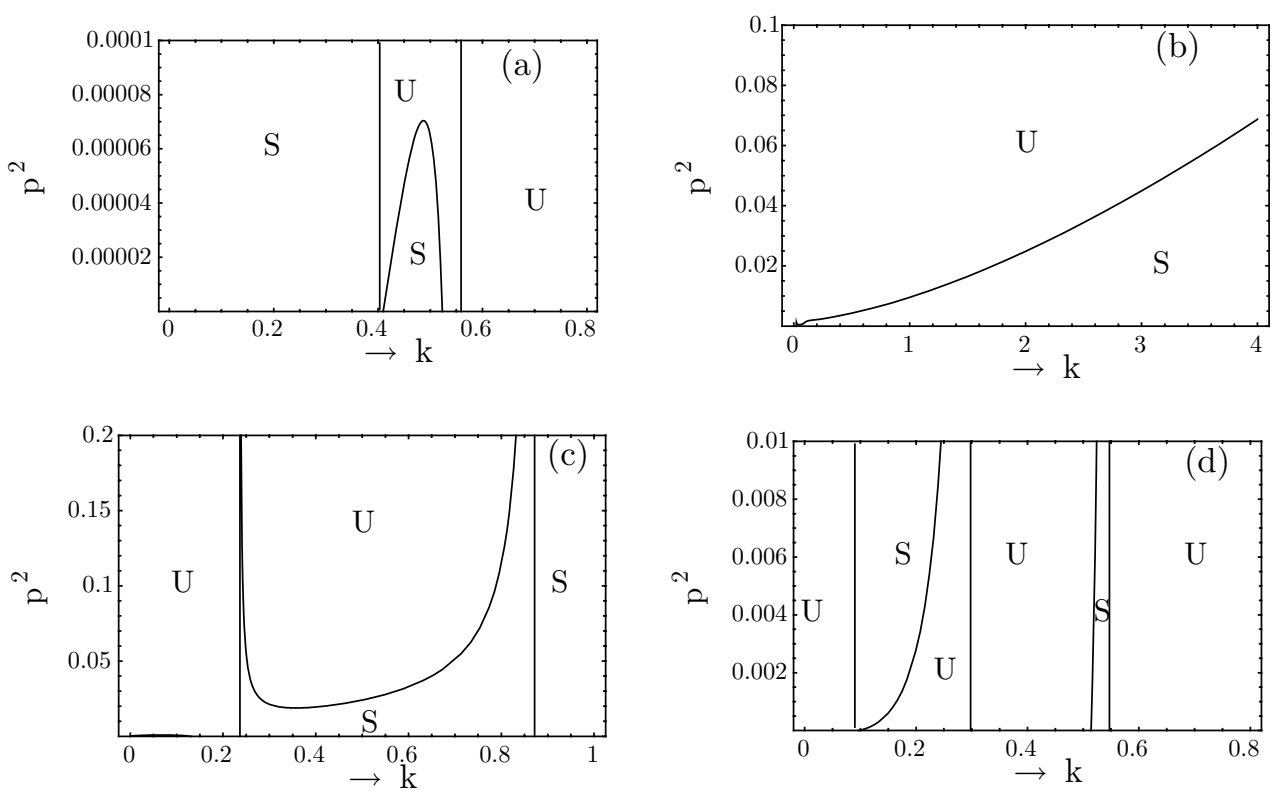

Fig. 5. For the same system considered in Fig. 3 but for $\rho=0.8$ and $u_{c 2}=0$.
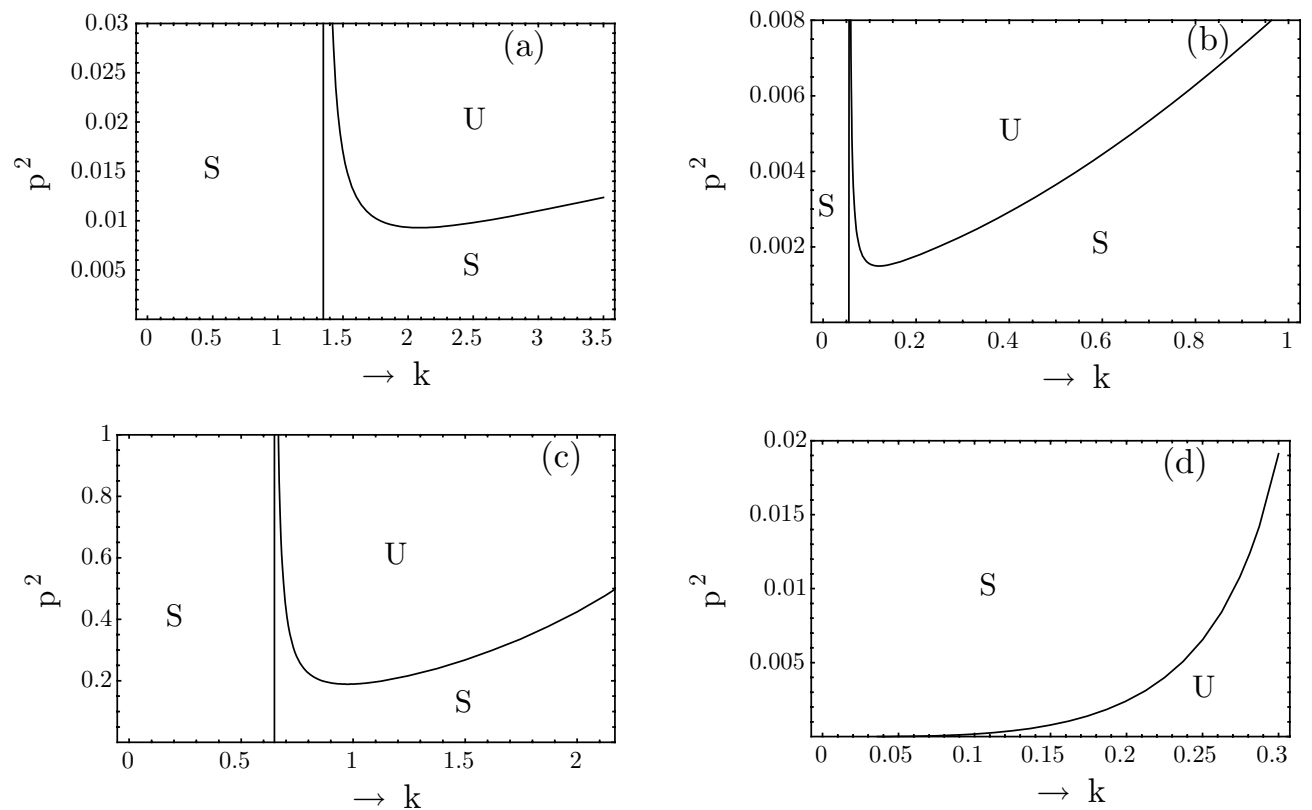

Fig. 6. For the same system considered in Fig. 3 but for $\rho=0.8$ and $u_{c 2}=2.6$. 
In part (a) of Fig. 4 we note that there is no essential change in the system's stability compared to the part in Fig. 3. We remark in Fig. 4(b) that the $\left(p^{2}, k\right)$ plane is divided by two branches of the transition curves into three areas, two of them stable and one unstable, while in Fig. 4(c) the system changed completely into a stable case, whereas we got in Fig. 4(d) three branches of the transition curves which divide the $\left(p^{2}, k\right)$ plane into four areas, three of them unstable and one stable. We see in the four parts of Fig. 5, in which $\rho=0.8, u_{c 2}=0$, that there is a qualitative change in the system's behavior through the number of the branches of the transition curves and also, the stable and unstable areas. We see in the different parts of Fig. 6 that $\rho=0.8$ and $u_{c 2}=2.6$, and there is a regular change to some extent with the change of the values of the viscosity numbers of the liquids compared with the change in the system's behavior as in the states which are presented by Figs. 3, 4, and 5. Hence, we obtained in part (a) of Fig. 6 one unstable area and two stable areas, and with the increase of the values of viscosity to make $\hat{\mu}_{\ell 1}=\hat{\mu}_{\ell 2}=2.02$ in part (b) of Fig. 6 , we see a decrease in the stable area which is concerned with the range $k<1.23$ in part (a) of the same figure to become the area which is concerned with the range $k<0.06$, while there is an increase in the other two areas meaning that there is a dislodgment in all the curves of Fig. 6(a) towards the left with an increase in the values of the two viscosity numbers. In Fig. 6(c), we consider an inviscid lower liquid by taking $\hat{\mu}_{\ell 2}=2.02$. In this case we note that the curves dislodge to the right to get a stable area concerned with the range $k<0.65$, while the range $k>0.65$ is divided into two areas. In part (d) of Fig. 6 , the above liquid is inviscid, while the lower has $\hat{\mu}_{\ell 1}=2.02$. The $\left(p^{2}, k\right)$ plane is divided by one curve into two areas, one of them is stable at the left of the curve whereas the other is unstable at the right, and we also see that the increase of the wavenumber augments the unstable area.

Modulating the trivial solution of equation (19), we obtain the following stability conditions:

$$
k \gamma_{i}\left(\hat{\mu}_{\ell 1}+\hat{\mu}_{\ell 1}\right)^{2}<\left(u_{c 2}-u_{c 1}\right)\left(\rho \hat{\mu}_{\ell 1}-\hat{\mu}_{\ell 2}\right) \gamma_{r} .
$$

If $\rho \hat{\mu}_{\ell 1}>\hat{\mu}_{\ell 2}$ and $\gamma_{r}>0$, the condition can be rewritten as follows:

$$
\left(u_{c 2}-u_{c 1}\right)>\frac{k \gamma_{i}\left(\hat{\mu}_{\ell 1}+\hat{\mu}_{\ell 1}\right)^{2}}{\left(\rho \hat{\mu}_{\ell 1}-\hat{\mu}_{\ell 2}\right) \gamma_{r}},
$$

and if $\gamma_{r}<0$, we have

$$
\left(u_{c 2}-u_{c 1}\right)<\frac{k \gamma_{i}\left(\hat{\mu}_{\ell 1}+\hat{\mu}_{\ell 1}\right)^{2}}{\left(\rho \hat{\mu}_{\ell 1}-\hat{\mu}_{\ell 2}\right) \gamma_{r}} .
$$

5.2. Stability of the nontrivial solution. In this section, the stability of the nontrivial solution of the evolution equations (17) and (19) is examined. In this work, we confine our aim to the temporal solution, which is expressed as

$$
A(\zeta, \xi)=A_{0} e^{-i \varpi \zeta},
$$

where $A_{0}$ depends on neither $\zeta$ nor $\xi$, and which must be a real quantity. Substituting (27) into (17), we obtain the following values of $A_{0}$ and $\varpi$ :

$$
\left|A_{0}\right|^{2}=-\frac{x_{r 2}}{x_{r 3}}
$$



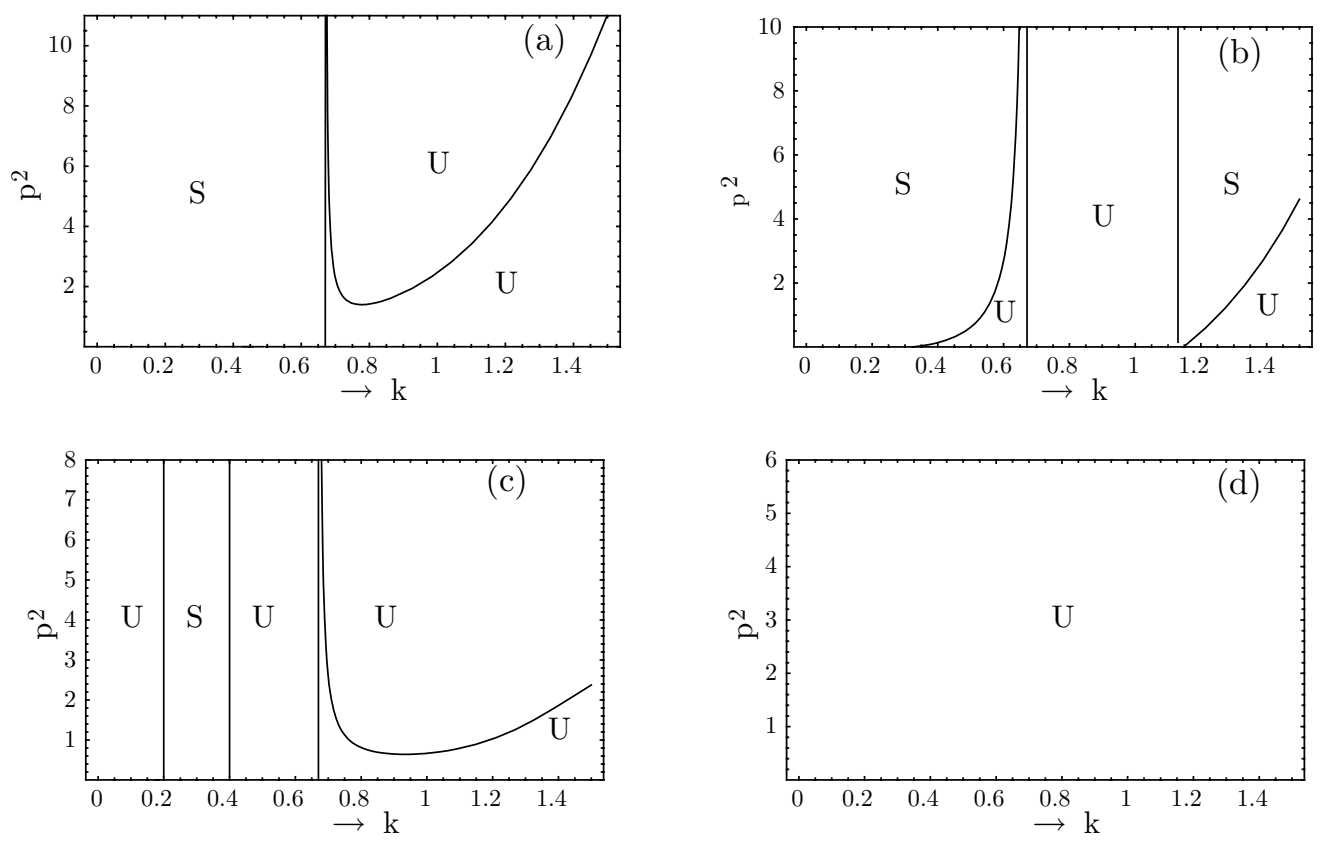

FIG. 7. The curves represent the conditions (32) and (33) for a system having $\rho=0.1$ and $u_{c 2}=0:$ (a) $\hat{\mu}_{\ell 1}=0.01$ and $\hat{\mu}_{\ell 2}=0.01$, (b) $\hat{\mu}_{\ell 1}=0.5$ and $\hat{\mu}_{\ell 2}=0.5$, (c) $\hat{\mu}_{\ell 1}=0.01$ and $\hat{\mu}_{\ell 2}=0.5$, (d) $\hat{\mu}_{\ell 1}=0.5$ and $\hat{\mu}_{\ell 2}=0.01$.

and

$$
\varpi=\frac{x_{i 2} x_{r 3}-x_{i 3} x_{r 2}}{x_{r 3}} .
$$

For the solution to exist, the right-hand side of (28) must be positive. This condition is expressed as $x_{r 2}<0$ and $x_{r 3}>0$, or $x_{r 2}>0$ and $x_{r 3}<0$. Assume that a small perturbation is superposed on the stated solution (27), to have

$$
A(\zeta, \xi)=\left(A_{0}+a_{1}(\zeta, \xi)\right) e^{i\left(-\varpi \zeta+b_{1}(\zeta, \xi)\right)},
$$

where $a_{1}(\zeta, \xi)$ and $b_{1}(\zeta, \xi)$ are real functions to be determined. Using the evolution equation (30), since the higher-order terms with respect to $a_{1}(\zeta, \xi)$ and $b_{1}(\zeta, \xi)$ are neglected, we obtain

$$
\begin{aligned}
& a_{1}(\zeta, \xi)=\hat{c}_{1} e^{i(\xi p-\hat{\varpi} \zeta)} \\
& b_{1}(\zeta, \xi)=\hat{c}_{2} e^{i(\xi p-\hat{\varpi} \zeta)},
\end{aligned}
$$

where $\hat{c}_{1}$ and $\hat{c}_{2}$ are real quantities and with $\hat{\varpi}$ satisfying the following dispersion relation:

$$
\begin{gathered}
\hat{\varpi}^{2}+c_{1} \hat{\varpi}+c_{2}=0, \\
c_{1}=-2 p^{2} x_{r 1}+2 A_{0}^{2} x_{r 3}, \\
c_{2}=p^{4} x_{i 1}^{2}-2 A_{0}^{2} p^{2} x_{i 1} x_{i 3}+p^{4} x_{r 1}^{2}-2 A_{0}^{2} p^{2} x_{r 1} x_{r 3} .
\end{gathered}
$$



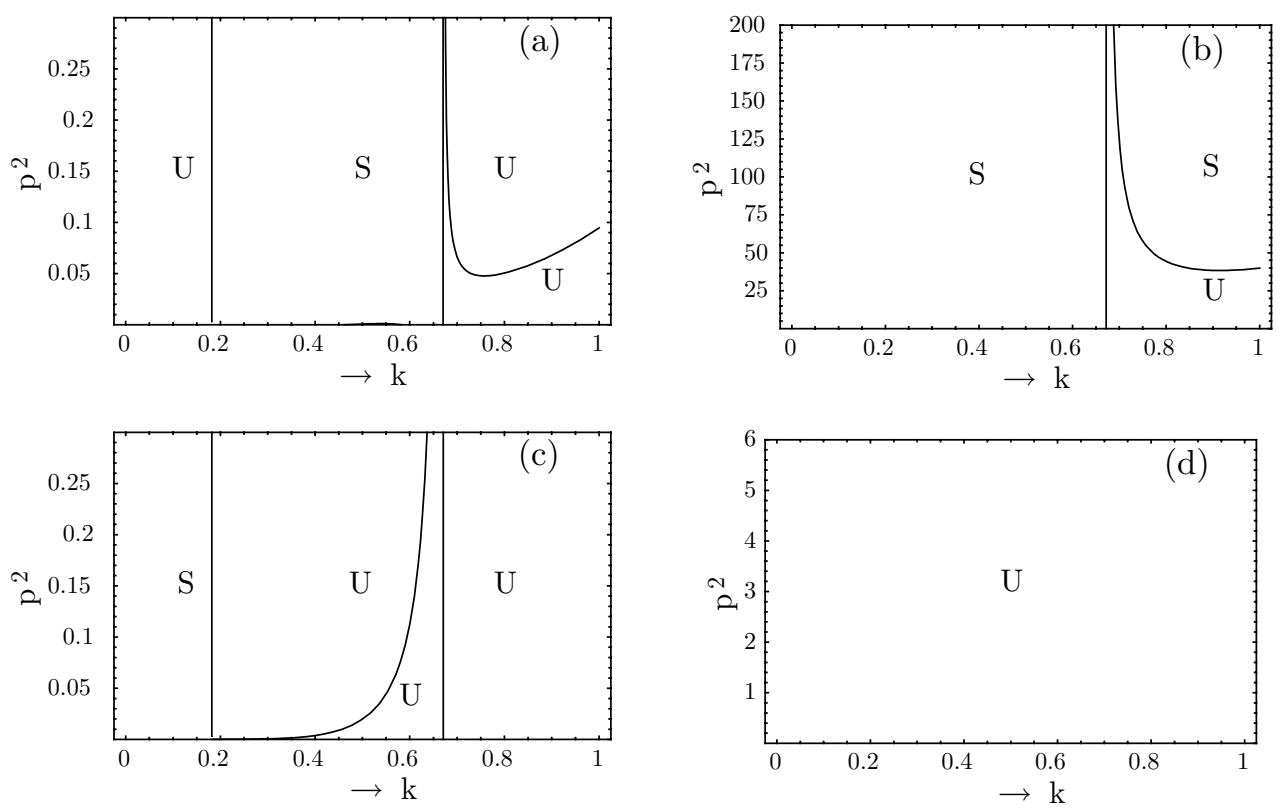

Fig. 8. For the same system considered in Fig. 7 but for $\rho=0.1$ and $u_{c 2}=1$.

The condition that the real part of $\hat{\varpi}$ is negative can be expressed as

$$
-2 p^{2} x_{r 1}+2 A_{0}^{2} x_{r 3}>0
$$

and

$$
\begin{gathered}
\left(x_{r 1}+4 x_{i 1} x_{r 1}\right) p^{6}+\left(16 x_{r 1} x_{r 2}+8 x_{i 1} x_{r 1} x_{r 2}+\frac{8 x_{i 1} x_{i 3} x_{r 1} x_{r 2}}{x_{r 3}}\right) p^{4} \\
+\left(4 x_{i 1} x_{r 2}+20 x_{r 1} x_{r 2}+\frac{16 x_{i 1} x_{i 3} x_{r 1} x_{r 2}}{x_{r 3}}\right) p^{2}+8 x_{r 1} x_{r 2}+\frac{8 x_{i 1} x_{i 3} x_{r 2}}{x_{r 3}} \geq 0 .
\end{gathered}
$$

The influence of the two viscosity coefficients on the stability criteria corresponding to the finite solution near the marginal state may be clarified by studying the stability graphs represented by the conditions (33) and $(34)$ in the $\left(p^{2}, k\right)$ the plane. The curves displayed in the parts of Fig. 7 correspond to a system having the physical parameters $\rho=0.1, u_{c 2}=0$. However, part (a) is drawn at $\hat{\mu}_{\ell 1}=\hat{\mu}_{\ell 2}=0.1$, part (b) drawn at $\hat{\mu}_{\ell 1}=\hat{\mu}_{\ell 2}=0.5$, part (c) drawn at $\hat{\mu}_{\ell 1}=0.01, \hat{\mu}_{\ell 2}=0.5$, while part (d) is drawn at $\hat{\mu}_{\ell 1}=0.5, \hat{\mu}_{\ell 2}=0.01$. Inspection of part (a) shows that there is only one transition curve which divides the stability plane into two regions: one of them is stable $(\mathrm{S})$ and lies above the transition curve while the other is unstable (U) and lies below that curve. In part 7 (b) we observe that the stability conditions produce two distinct transition curves that separate the stability plane into four regions: two of them are stable, while the others are unstable. It is observed that the system is stable for the long surface waves characterized by a wave number less than 2.8 , while the short waves are unstable. In part 7 (c), we observe that the stable region is slightly affected by the increase in the wave number in the specific range $0<k<0.9$, while this region is regularly increased with an increase 
in the wave number for $k>1$. On the other hand, in part $7(\mathrm{~d})$, we remark that a great change in the stability picture has occurred compared with part $7(\mathrm{c})$. It is observed that there is a regular decrease in the stable region in the specific range $0 \leq k<0.82$, while all the waves having wave number greater than 0.82 are stable. In the parts of Fig. 8 , we clarify the effects of the viscosity coefficients on the stability criteria of the surface waves for the same cases, respectively in Fig. 7 when the influence of the critical velocity $u_{c 2}=1$ of the upper fluid is presented.

In part 8(a), we have observed that the transition curves have divided the stability plane into two regions, one of them stable (the region below the curve), and the other unstable (the region above the curve). This means that the effect of $u_{c 2}$ is to change the stable region into an unstable one and vice versa. In part $8(\mathrm{~b})$, we notice that the behavior of the stability picture is different from that of part 7(a), where there is only a stable region corresponding to the long waves, while the remaining waves are unstable. On comparing the parts $8(\mathrm{c}), 6(\mathrm{c})$ to each other, we have noticed that the behaviors of the stability criteria of each case are very similar. On the other hand, in part $8(\mathrm{~d})$, we observe that there is a great change in the stability behavior of the system compared to the case of $7(\mathrm{~d})$, where the unstable region has been changed to a stable one and then we have a global stability case whatever the $k$ value.

On the marginal curve, and using the the modulation concept, the stability conditions of the waves are given as follows:

$$
k \gamma_{i}\left(\hat{\mu}_{\ell 1}+\hat{\mu}_{\ell 1}\right)^{2}<\left(u_{c 2}-u_{c 1}\right)\left(\rho \hat{\mu}_{\ell 1}-\hat{\mu}_{\ell 2}\right) \gamma_{r}
$$

and

$$
p^{2}>-\frac{2 a^{2} \beta_{r} x_{i 1}}{x_{i 1}^{2}+x_{r 1}^{2}}
$$

where $a^{2}$ is a real constant and $p$ is the wavenumber of the modulations. When $\beta_{r} x_{i 1}>0$, the condition (36) will be trivial and thereby the condition (35) will be the only condition of stability. In this case the trivial solution and the nontrivial solution have the same stability condition (35) at the marginal state.

The graphs displayed in the sections of Fig. 9 represent the transition curve for the stability conditions (35) and (36), which correspond to the exact marginal state. In the parts of this figure, we examine the effect of the viscosity coefficients on the stability behavior of the system for the same values of $\hat{\mu}_{\ell 1}, \hat{\mu}_{\ell 2}$ that have been taken in the parts of Fig. 7, respectively, when $u_{c 1} \neq 0, u_{c 2}=0$. An investigation of part 9(a) shows that the stability conditions are satisfied and hence the surface waves are stable in the range $0 \leq k \leq 0.42$, while the remaining waves are unstable; while in part $9(\mathrm{~b})$, we observe that the surface waves are stable in the two regions $0 \leq k \leq 0.45$, and $2.38 \leq k$, whereas these waves are unstable as $0.45 \leq k<2.38$. Moreover, the graph of part 9 (c) shows that the stability plane still needs to be divided into three regions: two of them, characterized by $0 \leq k \leq 0.21$ and $2.2 \leq k$, are surface waves having the stability conditions, while the third one, characterized by $0.21<k<2.2$, is a surface wave lacking such conditions. In part $9(\mathrm{~d})$, we have stable surface waves corresponding to the regions $0 \leq k \leq 0.23$ and $4.02 \leq k \leq 6.1$, while the surface waves corresponding to the regions $0.23<k<4.02$ and $k>6.1$ are unstable. In the sections of Fig. 10, we repeat 

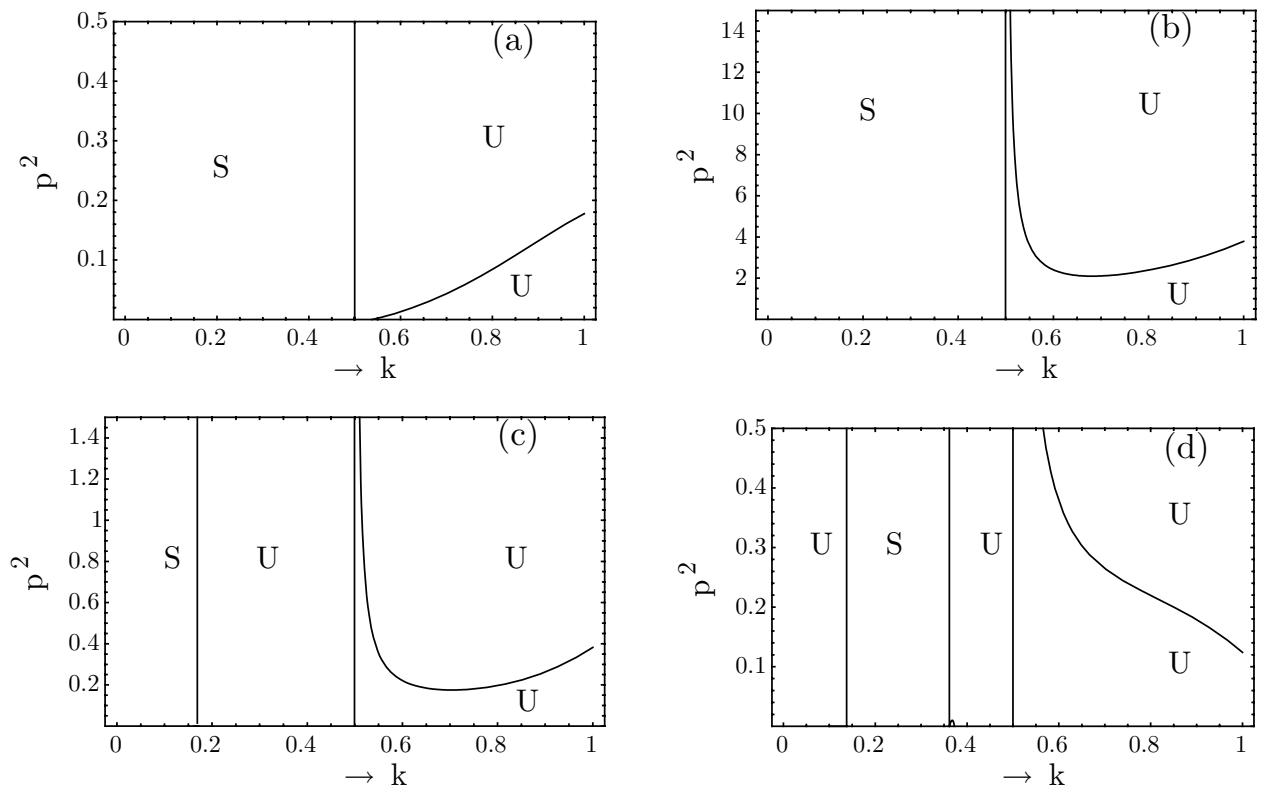

FIG. 9. The curves represent the conditions (32) and (33) for a system having $\rho=0.1$ and $u_{c 2}=0$ : (a) $\hat{\mu}_{\ell 1}=0.01$ and $\hat{\mu}_{\ell 2}=0.01$, (b) $\hat{\mu}_{\ell 1}=0.5$ and $\hat{\mu}_{\ell 2}=0.5$, (c) $\hat{\mu}_{\ell 1}=0.01$ and $\hat{\mu}_{\ell 2}=0.5$, (d) $\hat{\mu}_{\ell 1}=0.5$ and $\hat{\mu}_{\ell 2}=0.01$.
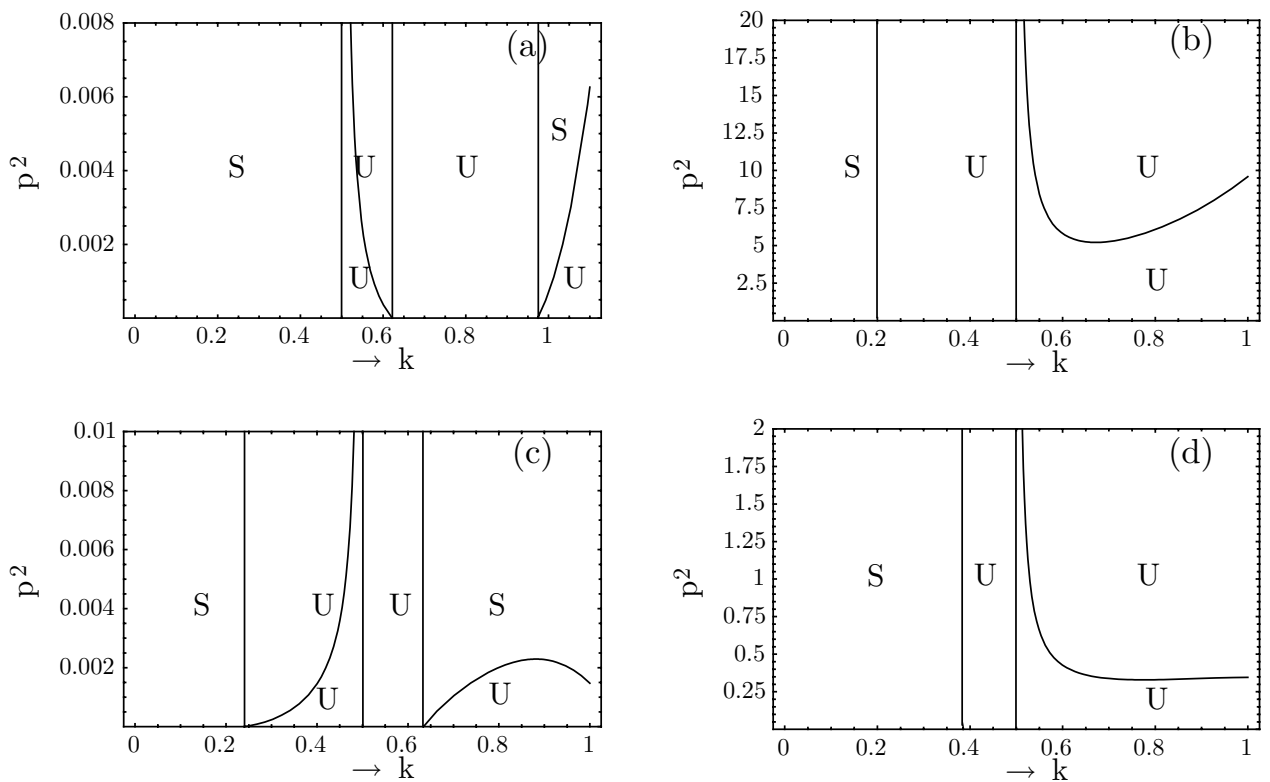

FIG. 10. For the same system considered in Fig. 9 but for $\rho=0.1$ and $u_{c 2}=1$. 
the same calculations depicted in the parts of Fig. 9, respectively, but when $u_{c 2}=0$. Generally, we have observed that there is a great change in the stability behavior of the system when Fig. 9 and Fig. 10 are compared to each other. However, the graphs displayed in part 10(a) indicate that only the surface waves corresponding to the region $0 \leq k \leq 0.4$ are stable, while the other waves are unstable. Further, in part 10(b) we have a globally stable surface wave. In part 10(c), we observe that the system is still globally stable, while in part 10(d) the most stable waves are changed into unstable waves and we have stable waves only through the specific range $0 \leq k \leq 0.3$, approximately.

6. Conclusion. According to the linear step of the problem, we see clearly that the increase in the $\rho$ value plays an important role in determining the influence of the viscosity numbers of the two liquids on the stability system in linear theory, but this role will disappear completely when the values of the viscosity numbers are equal. On the other hand, the two viscosity numbers play a dual role in that they sometimes play a stable role while in other cases an unstable role depending on the $\rho$ values.

From studying the trivial solution near the marginal state, we note that the behavior of the modulation of the trivial solutions near the marginal state was exposed to noticeable changes with the change of the viscosity numbers altogether although these two values are equal unlike in the linear state, and also the increase in the value of the viscosity number of any of the liquids, including the lack of the same value for the number of the other liquid, has an irregular effect on the nonlinear step and this role is determined according to the values of the density and the stream velocity.

From studying the nontrivial solution near (on) the marginal state, we remark that the increase (decrease) of viscosity numbers plays a dual role on the stability criteria of the interfacial waves. The increase in the values of the speed of the flow leads to the enrichment of the numbers of unstable waves.

Appendix. Order of $\varepsilon$ :

$$
\begin{gathered}
\partial_{x_{0}}^{2} \phi_{j 1}+\partial_{y}^{2} \phi_{j 1}=0 \\
\partial_{y} \phi_{j 1}-\partial_{t_{0}} \eta_{1}-u_{j} \partial_{x_{0}} \eta_{1}=0 \\
\sum_{j=1}^{2}(-\rho)^{j-1}\left(\partial_{t_{0}} \phi_{j 1}+u_{j} \partial_{x_{0}} \phi_{j 1}\right)+2(-1)^{j-1} \hat{\mu}_{\ell j} \partial_{y}^{2} \phi_{j 1}+(1-\rho) \eta_{1}-\partial_{x_{0}}^{2} \eta_{1}=0 .
\end{gathered}
$$


Order of $\varepsilon^{2}$ :

$$
\begin{gathered}
\partial_{x_{0}}^{2} \phi_{j 2}+\partial_{y}^{2} \phi_{j 2}=-2 \partial_{x_{1}} \partial_{x_{0}} \phi_{j 1} \\
\partial_{y} \phi_{j 2}-\partial_{t_{0}} \eta_{2}-u_{j} \partial_{x_{0}} \eta_{2}=\partial_{t_{1}} \eta_{1}+u_{j} \partial_{x_{1}} \eta_{1}+\partial_{x_{0}} \eta_{1} \partial_{x_{0}} \phi_{j 1}-\eta_{1} \partial_{y}^{2} \phi_{j 1} \\
\sum_{j=1}^{2}(-\rho)^{j-1}\left(\partial_{t_{0}} \phi_{j 2}+u_{j} \partial_{x_{0}} \phi_{j 2}\right)+2(-1)^{j-1} \hat{\mu}_{\ell j} \partial_{y}^{2} \phi_{j 2}+(1-\rho) \eta_{2}-\partial_{x_{0}}^{2} \eta_{2} \\
=\sum_{j=1}^{2}(-1)^{j}(\rho)^{j-1}\left(\partial_{t_{1}} \phi_{j 1}+\eta_{1} \partial_{t_{0}} \partial_{y} \phi_{j 1}+u_{j} \partial_{x_{1}} \phi_{j 1}+\frac{1}{2}\left(\partial_{x_{0}} \phi_{j 1}\right)^{2}+\frac{1}{2}\left(\partial_{y} \phi_{j 1}\right)^{2}\right. \\
\left.+u_{j} \eta_{1} \partial_{x_{0}} \partial_{y} \phi_{j 1}\right)+(-1)^{j} \hat{\mu}_{\ell j}\left(2 \eta_{1} \partial_{y}^{3} \phi_{j 1}-4 \partial_{x_{0}} \eta_{1} \partial_{x_{0}} \partial_{y} \phi_{j 1}\right)-2 \partial_{x_{0}} \partial_{x_{1}} \eta_{1} .
\end{gathered}
$$

Order of $\varepsilon^{3}$ :

$$
\begin{aligned}
& \partial_{x_{0}}^{2} \phi_{j 3}+\partial_{y}^{2} \phi_{j 3}=-2 \partial_{x_{1}} \partial_{x_{0}} \phi_{j 2}-\partial_{x_{1}}^{2} \phi_{j 1} \\
& \partial_{y} \phi_{j 3}-\partial_{t_{0}} \eta_{3}-u_{j} \partial_{x_{0}} \eta_{3}=\partial_{t_{1}} \eta_{2}+\partial_{t_{2}} \eta_{1}+u_{j} \partial_{x_{1}} \eta_{2}+\partial_{x_{0}} \eta_{1} \partial_{x_{0}} \phi_{j 2} \\
& +\partial_{x_{0}} \eta_{2} \partial_{x_{0}} \phi_{j 1}+\partial_{x_{1}} \eta_{1} \partial_{x_{0}} \phi_{j 1}+\partial_{x_{0}} \eta_{1} \partial_{x_{1}} \phi_{j 1}+\eta_{1} \partial_{x_{0}} \eta_{1} \partial_{x_{0}} \partial_{y} \phi_{j 1} \\
& -\eta_{2} \partial_{y}^{2} \phi_{j 1}-\eta_{1} \partial_{y}^{2} \phi_{j 2}-\frac{1}{2} \eta_{1}^{2} \partial_{y}^{3} \phi_{j 1} \\
& \sum_{j=1}^{2}(-\rho)^{j-1}\left(\partial_{t_{0}} \phi_{j 3}+u_{j} \partial_{x_{0}} \phi_{j 3}\right)+2(-1)^{j-1} \hat{\mu}_{\ell j} \partial_{y}^{2} \phi_{j 3}+(1-\rho) \eta_{3}-\partial_{x_{0}}^{2} \eta_{3} \\
& =\sum_{j=1}^{2}(-1)^{j}(\rho)^{j-1}\left(\partial_{t_{1}} \phi_{j 2}+\partial_{t_{2}} \phi_{j 1}+\eta_{2} \partial_{t_{0}} \partial_{y} \phi_{j 1}+\eta_{1} \partial_{t_{0}} \partial_{y} \phi_{j 2}+\eta_{1} \partial_{t_{1}} \partial_{y} \phi_{j 1}\right. \\
& +\frac{1}{2} \eta_{1}^{2} \partial_{t_{0}} \partial_{y}^{2} \phi_{j 1}+u_{j}\left(\partial_{x_{1}} \phi_{j 2}+\eta_{1} \partial_{x_{1}} \partial_{y} \phi_{j 1}+\eta_{2} \partial_{x_{0}} \partial_{y} \phi_{j 1}+\eta_{1} \partial_{x_{0}} \partial_{y} \phi_{j 2}\right. \\
& \left.+\frac{1}{2} \eta_{1}^{2} \partial_{x_{0}} \partial_{y}^{2} \phi_{j 1}\right)+\partial_{x_{0}} \phi_{j 1} \partial_{x_{0}} \phi_{j 2}+\partial_{x_{1}} \phi_{j 1} \partial_{x_{0}} \phi_{j 1}+\partial_{y} \phi_{j 1} \partial_{y} \phi_{j 2} \\
& +\eta_{1} \partial_{y} \phi_{j 1} \partial_{y}^{2} \phi_{j 1}+\eta_{1} \partial_{x_{0}} \phi_{j 1} \partial_{x_{0}} \partial_{y} \phi_{j 1} \\
& +(-1)^{j} \hat{\mu}_{\ell j}\left(2 \eta_{1} \partial_{y}^{3} \phi_{j 2}+2 \eta_{2} \partial_{y}^{3} \phi_{j 1}-4 \partial_{x_{0}} \eta_{1} \partial_{x_{0}} \partial_{y} \phi_{j 2}-4 \partial_{x_{0}} \eta_{2} \partial_{x_{0}} \partial_{y} \phi_{j 1}\right. \\
& -4 \partial_{x_{1}} \eta_{1} \partial_{x_{0}} \partial_{y} \phi_{j 1}-4 \partial_{x_{0}} \eta_{1} \partial_{x_{1}} \partial_{y} \phi_{j 1}+2\left(\partial_{x_{0}} \eta_{1}\right)^{2} \partial_{x_{0}}^{2} \phi_{j 1}-2\left(\partial_{x_{0}} \eta_{1}\right)^{2} \partial_{y}^{2} \phi_{j 1} \\
& \left.-4 \eta_{1} \partial_{x_{0}} \eta_{1} \partial_{y}^{2} \phi_{j 1}+\eta_{1}^{2} \partial_{y}^{4} \phi_{j 1}\right)+\partial_{x_{1}}^{2} \eta_{1}+2 \partial_{x_{0}} \partial_{x_{1}} \eta_{2}-\frac{3}{2}\left(\partial_{x_{0}} \eta_{1}\right)^{2} \partial_{x_{0}}^{2} \eta_{1}, \\
& \lambda_{r 1}=-\frac{2 \omega(1+\rho)}{k}+2 u_{c 1}+2 \rho u_{c 2}, \lambda_{i 1}=-2 k\left(\hat{\mu}_{\ell 1}+\hat{\mu}_{\ell 2}\right), \\
& \lambda_{r 2}=-k-\frac{\omega^{2}}{k^{2}}(1+\rho)+u_{c 1}^{2}+\rho u_{c 2}, \\
& \lambda_{i 2}=2 \omega^{2}\left(\hat{\mu}_{\ell 1}+\hat{\mu}_{\ell 2}\right)-4 k\left(u_{c 1} \hat{\mu}_{\ell 1}+u_{c 2} \hat{\mu}_{\ell 2}\right), \quad \lambda_{3}=\left(\omega-k u_{c 1}\right) \Delta_{1}+\left(\omega-k u_{c 2}\right) \Delta_{2}, \\
& \gamma_{r}=-2 \frac{(1+\rho) \lambda_{i 2} \lambda_{i 1}^{2} \lambda_{r 2}}{k\left(\lambda_{i 1}^{2}+\lambda_{r 1}^{2}\right)^{2}}+2 \frac{(1+\rho) \lambda_{i 2}^{2} \lambda_{i 1} \lambda_{r 1}}{k\left(\lambda_{i 1}^{2}+\lambda_{r 1}^{2}\right)^{2}}-2 \frac{(1+\rho) \lambda_{r 2}^{2} \lambda_{i 1} \lambda_{r 1}}{k\left(\lambda_{i 1}^{2}+\lambda_{r 1}^{2}\right)^{2}} \\
& +2 \frac{(1+\rho) \lambda_{i 2} \lambda_{r 2} \lambda_{r 1}^{2}}{k\left(\lambda_{i 1}^{2}+\lambda_{r 1}^{2}\right)^{2}}+2 \frac{\omega(1+\rho) \lambda_{i 1} \lambda_{r 2}}{k^{2}\left(\lambda_{i 1}^{2}+\lambda_{r 1}^{2}\right)}-2 \frac{\omega(1+\rho) \lambda_{r 1} \lambda_{r 2}}{k^{2}\left(\lambda_{i 1}^{2}+\lambda_{r 1}^{2}\right)}
\end{aligned}
$$




$$
\begin{gathered}
+2 \frac{\left(\hat{\mu}_{\ell 1}+\hat{\mu}_{\ell 2}\right) \lambda_{i 1} \lambda_{r 2}}{\lambda_{i 1}^{2}+\lambda_{r 1}^{2}}+2 \frac{\left(\hat{\mu}_{\ell 1}+\hat{\mu}_{\ell 2}\right) \lambda_{r 1} \lambda_{r 2}}{\lambda_{i 1}^{2}+\lambda_{r 1}^{2}}-2 u_{c 1} \hat{\mu}_{\ell 1}-2 u_{c 2} \hat{\mu}_{\ell 2}, \\
\gamma_{i}=1-\frac{\omega^{2}(1+\rho)}{k^{3}}-\frac{(1+\rho) \lambda_{i 2}^{2} \lambda_{i 1}^{2}}{k\left(\lambda_{i 1}^{2}+\lambda_{r 1}^{2}\right)^{2}}+\frac{(1+\rho) \lambda_{i 1}^{2} \lambda_{r 2}^{2}}{k\left(\lambda_{i 1}^{2}+\lambda_{r 1}^{2}\right)^{2}}-4 \frac{(1+\rho) \lambda_{r 2} \lambda_{i 1} \lambda_{r 1} \lambda_{i 2}}{k\left(\lambda_{i 1}^{2}+\lambda_{r 1}^{2}\right)^{2}} \\
+\frac{(1+\rho) \lambda_{i 2}^{2} \lambda_{r 1}^{2}}{k\left(\lambda_{i 1}^{2}+\lambda_{r 1}^{2}\right)^{2}}-\frac{(1+\rho) \lambda_{r 2}^{2} \lambda_{r 1}^{2}}{k\left(\lambda_{i 1}^{2}+\lambda_{r 1}^{2}\right)^{2}}+2 \frac{\omega(1+\rho) \lambda_{i 1} \lambda_{r 2}}{k^{2}\left(\lambda_{i 1}^{2}+\lambda_{r 1}^{2}\right)} \\
\lambda_{r 1}^{2} \lambda_{i 1} \lambda_{r 1}+\lambda_{r 1}^{2} \\
\beta_{r}=2 \omega^{2}(1-\rho) Q_{i 1}^{2}-4 k \omega Q_{i 1} u_{c 1}+2 k^{2} Q_{i 1} u_{c 1}^{2}+4 k \rho \omega Q_{i 1} u_{c 2}-2 k^{2} \rho Q_{i 1} u_{c 2} \\
\quad+6 k^{3} \omega \hat{\mu}_{\ell 1}-6 k^{4} u_{c 1} \hat{\mu}_{\ell 1}+6 k^{3} \omega \hat{\mu}_{\ell 2}-6 k^{4} u_{c 2} \hat{\mu}_{\ell 2}, \\
\beta_{i}=\frac{3}{2} k^{4}-2 k \omega^{2}(1+\rho)-2 \omega^{2} Q_{r 1}(1-\rho)+4 k^{2} \omega u_{c 1}+4 k \omega Q_{r 1} u_{c 1}-2 k^{3} u_{c 1}^{2} \\
-2 k^{2} Q_{r 1} u_{c 1}^{2}+4 k^{2} \rho \omega u_{c 2}-4 k \rho \omega Q_{r 1} u_{c 2}-2 k^{3} \rho u_{c 2}^{2}+2 k^{2} \rho Q_{r 1} u_{c 2}^{2} .
\end{gathered}
$$

\section{REFERENCES}

[1] D.D. Joseph and T. Liao, Potential flows of viscous and viscoelastic fluids. J. Fluid Mech. 265 (1994), 1-23. MR1271678 (95e:76032)

[2] D.D. Joseph and T. Liao, Viscous and viscoelastic potential flow, Trends and Perspectives in Appl. Math., Appl. Math. Science 100, Springer-Verlag (1994), 1-54. MR.1277194(95h:76002)

[3] D.D. Joseph, J. Belanger and G.S. Beavers, Breakup of a liquid drop suddenly exposed to a highspeed air stream. Int. J. Multiphase Flow 25 (1999), 1263-1303.

[4] T. Funada and D.D. Joseph, Viscous potential flow analysis of Kelvin-Helmholtz instability in a channel, J. Fluid Mech. 445 (2001), 263-283. MR1875700(2002k:76060)

[5] D.D. Joseph, Viscous potential flow, J. Fluid Mech. 479 (2003), 191-197. MR2011824|(2004i:76163)

[6] D.D. Joseph and J. Wang, The dissipation approximation and viscous potential flow, J. Fluid Mech. 505 (2004), 365-377. MR2259003 (2007d:76099)

[7] J. Wang and D.D. Joseph, Pressure corrections for the effects of viscosity on the irrotational flow outside Prandtl's boundary layer, J. Fluid Mech. 557 (2006), 145-165. MR.2265518 (2007g:76064)

[8] H. Hasimoto and H. Ono, Nonlinear modulation of gravity waves, J. Phy. Soc. Japan 33 (1972), 805-811.

[9] A.H. Nayfeh, Nonlinear propagation of wave packets on fluid interface, Trans. ASME 98E (1976), 584-588.

[10] P.K. Newton and J.B. Keller, Stability of plane wave solutions of nonlinear systems, Wave Motion 10 (1988), 183-191. MR934920(89e:76021)

[11] K. Zakaria, Nonlinear dynamics of magnetic fluids with a relative motion in the presence of an oblique magnetic field, Physica A, 327 (2003), 221-248. MR2007131(2004h:76170)

[12] K. Zakaria, Wilton ripples between two uniform streaming magnetic fluids, Int. J. of Nonlinear Mech. 39 (2004), 1051-66. 